\title{
Some studies on inherited disease using the techniques of cell culture
}

\author{
A. G. BeARN \\ Department of Medicine, Cornell University Medical College, \\ and The New York Hospital
}

IF ONE CAN JUDGE from recent trends in Departments of Medicine throughout the world, it would seem that the future of medical research lies in an increasingly reductionist approach to human disease. Indeed, in some of the most productive departments, the direct study of human disease is frequently accorded less attention, and certainly less acclaim, than studies on individual cells and enzymes, often derived from other species. This trend, which clearly had its origins in the spectacular success of molecular biology, ignores the lessons learned by the pioneering clinical scientists who, by their teaching and research, have amply demonstrated that direct studies on man can contribute brilliantly to basic scientific knowledge, and at the same time benefit mankind. The isolation, characterization and crystallization of a rat liver enzyme, superficially so scientifically elegant, is frequently mere hackwork compared to the performance of imaginative, meaningful clinical investigation. It is worth recalling that the discovery of DNA as the transforming principle had its origin at the bedside where physicians were concerned not with elucidating the code of life, but with the preparation of a suitable antiserum to treat patients with pneumococcal pneumonia. The clinical scientist is continually bedevilled by the complexity of homeostatic mechanisms so important for man's survival, but which too often obscure the results, delight the statistician, and frustrate the investigator. Yet, many important areas of human biology remain to be explored, and discoveries are still to be made, by refocussing directly on human disease.

\section{Hurler's syndrome}

During the past 5 years, my colleagues and I have been investigating certain inherited storage diseases of mucopolysaccharide metabolism. It is a happy circumstance that enables me to relate that, like many good things, the condition now commonly known as Hurler's syndrome had its birth in Scotland. It was first recognized as a disease entity in 1900 by Dr John Thompson at the Royal Infirmary in Edinburgh, who felicitously called it 'Johnnie McL's disease' (Henderson, 1940). Your contributor could not uncover whether, as he naturally suspects, it was for convenience abbreviated to 'Mac's disease'.

Clinically, Hurler's syndrome is characterized by dwarfism, grotesque skeletal deformities, mental retardation, deafness, joint contractures, hepatosplenomegaly, and clouding of the cornea. Cardiac murmurs and valvular heart disease have long been recognized (McKusick, 1966). Our studies with this disease have centered on attempts to identify healthy carriers of the abnormal gene, and to seek ways in which the primary phenotypic abnormality - excessive mucopolysaccharide accumulation in the tissues -could be alleviated (Danes \& Bearn, 1966a, b).

There are several forms of Hurler's syndrome, which differ clinically, but unfortunately sharp distinction between different syndromes cannot always be made. Family studies have shown that there are two inherited varieties of Hurler's syndrome-one caused by a gene on an autosome and the other by a gene on the $\mathrm{X}$-chromosome. The autosomal variety is recessive-that is, two genes, one on each of the two homologous chromosomes, are necessary for the disease to manifest itself. The $\mathrm{X}$-linked variety is also recessive: a boy manifests the disease if the gene is present on his single $\mathbf{X}$ chromosome, whereas a girl, if the abnormal gene is present on only one of her X chromosomes, is a symptomfree carrier of the gene. Although the primary inherited defect in Hurler's syndrome remains to be elucidated, a constant feature of the disease is an increased cellular mucopolysaccharide content and, diagnostically most helpful, an increased urinary secretion of mucopolysaccharides, particularly chondroitin sulphate $\mathbf{B}$ and heparitin sulphate.

In an attempt to identify healthy carriers of the disease, it appeared worthwhile examining 
the urine to see if an increased excretion of mucopolysaccharides could be detected. We were disappointed to find that despite the use of fairly sophisticated chemical methods, no difference could be clearly established between normal subjects and those individuals known from pedigree studies to be carriers. Temporarily discouraged we tried a different approach. Since the tissues of the body in Hurler's syndrome are loaded with excess mucopolysaccharides, we argued that it might be possible to demonstrate an increased mucopolysaccharide in the skin of patients with the disease. Skin biopsies were obtained from normal subjects, patients with the disease, and certain of their relatives. Each skin biopsy was cultured and within a few weeks the tissue explants were surrounded by a halo of fibroblasts. These fibroblasts were then grown as a thin monolayer culture and stained with the metachromatic dye toluidine blue $O$, which stains mucopolysaccharides bright red. All the fibroblasts derived from affected individuals stained red indicating an increased mucopolysaccharide content, while those from normal subjects stained blue. More interesting (since there are easier ways of distinguishing normal individuals from patients with Hurler's syndrome than by culturing their fibroblasts), the healthy fibroblasts derived from those individuals who, from pedigree studies, were known to be carriers of the gene also stained bright red-revealing in a dramatic way the presence of the abnormal gene. It appeared likely that this simple method might allow us to define more clearly the two inherited types of Hurler's syndrome. An acid test presented itself when we were faced with an only child, a small boy aged 11, who had Hurler's syndrome, but in whom, because of insufficient pedigree data, we could not decide whether he had the autosomal or X-linked form of the disease. This distinction was of more than academic interest, because the probability of any subsequent son being affected would be $50 \%$ if the gene were on the $\mathrm{X}$ chromosome, and only $25 \%$ if it were on an autosome. Moreover, if the condition were $\mathrm{X}$-linked, $50 \%$ of all daughters would be healthy carriers of the gene who could bequeath the gene to the next generation. Both normal healthy parents agreed to have their skin cultured. The fibroblasts from the mother stained bright red with toluidine blue, indicating she was a carrier, whereas the skin derived from the father stained blue as do normal subjects; thus it could be concluded that the offending gene was clearly on one of the $X$ chromosomes of the patient's mother, who in turn had passed the gene on to her son. In patients in whom the disease is inherited as an autosomal recessive, the fibroblasts of both carrier parents stain bright red with toluidine blue. Thus it is possible to detect carriers of this disease by the simple expedient of growing the skin (Fig. 1). As a consequence, many sibs of affected individuals can be reassured that they are not carriers of the gene, and those healthy individuals who prove to be carriers can be given genetic counselling.

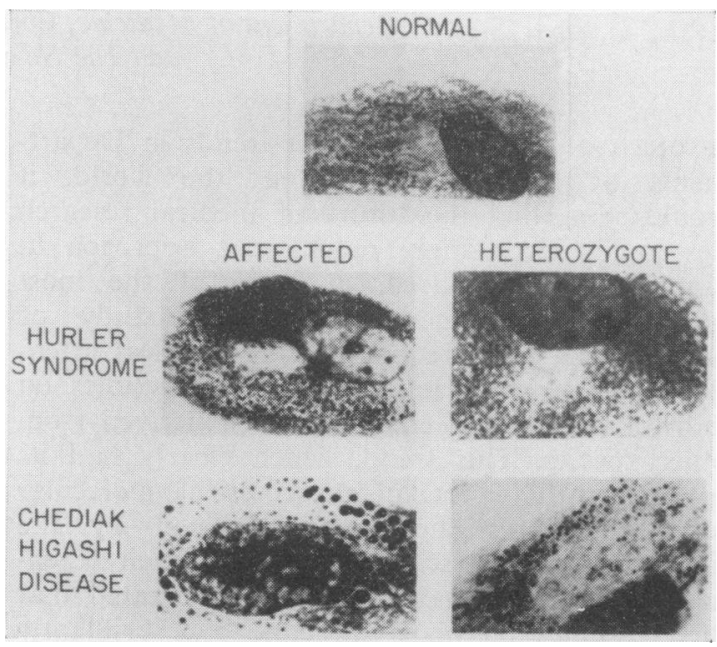

FIG. 1. Fibroblast cultures stained with toluidine blue $O$. The intracellular inclusions in Hurler's syndrome stain metachromatically and appear intensely red. In ChediakHigashi disease the inclusions are not metachromatic and have a slate-grey appearance.

\section{Chediak-Higashi disease}

Encouraged by our findings with the mucopolysaccharidoses, we turned to another inherited disease-Chediak-Higashi (White, 1966).

This autosomal recessively inherited disease is exceptionally rare, but the luminous example of Garrod and his studies on the inborn errors of metabolism persuaded us that this condition might yield information of general biological interest. The disease is characterized by decreased pigmentation of the skin, shifting nystagmus, and a peculiar susceptibility to infections. Unlike another experiment of nature, agammaglobulinaemia, the increased susceptibility to infections is not due to a deficient production of antibodies. However, quite recently, an abnormality of the blood cell series has been described. Within the cytoplasm of the granulocytes, large peroxidase-positive granules can be easily observed (Sato, 1955). These granules are large and frequently appear to coalesce. Evidence from 
the light microscope as well as from electron microscopic studies suggest they may represent large lysozomes. Interestingly, a very similar disease occurs in Aleutian mink, where it represents an economic anxiety. Mink breeders would much like to identify those apparently healthy mink which carry the gene for this disease to avoid the financial disaster of breeding two heterozygotes which would yield affected mink. The hope that carriers of the abnormal gene would demonstrate characteristic white cell inclusions, similar to those in affected individuals, proved unjustified and thus far no regular abnormality can be detected in the white blood cells of the heterozygous carriers.

However, encouraged by the findings with Hurler's syndrome, a skin biopsy was obtained from an affected child, both his parents, as well as control subjects. Using the same technique, fibroblast monolayer cultures were obtained and the cells stained with toluidine blue $O$ and Wright-Giemsa. It readily became apparent that the fibroblasts derived from the affected individual demonstrated discrete slate-grey ametachromatic cytoplasmic inclusions, similar to those seen in the peripheral white cells (Danes \& Bearn, 1967). The fibroblasts from the heterozygous father and mother did not differ from the normal controls during the first month in culture. During the second month, cell lines derived from both parents contained an occasional abnormal granule and by the close of the second month abnormal cytoplasmic granules were easily demonstrable in the cells derived from both parents. These studies need extending, but it seems likely that in Chediak-Higashi disease, as in Hurler's syndrome, the heterozygous state can be regularly detected using this technique (Fig. 1).

The demonstration of a phenotypic abnormality in the cultured fibroblasts in both Hurler's syndrome and the Chediak-Higashi syndrome raised the possibility that it might be feasible to utilize these in vitro systems to investigate the potential reversibility of the abnormal phenotype. It is commonly presumed that the abnormal signs of Hurler's syndrome are directly due to the increased mucopolysaccharide content of the cells. If the increased synthesis of mucopolysaccharides could be decreased in vitro the way would be open to investigate whether similar measures would exert an effect in vivo. Moreover, the demonstration of increased mucopolysaccharides in the fibroblasts of patients with this disease by metachromatic staining allows easy visual monitoring of the mucopolysaccharide content of the cell.

Fell and her colleagues at the Strangeways
Laboratory in Cambridge demonstrated in 1956 that when retinol (vitamin A) was added in vitro to embryonic cartilage, the cartilage was no longer able to become sulphated and this biochemical consequence was swiftly followed by a loss of cellular metachromasia. It was immediately apparent that it would be of considerable interest to see whether retinol would exert a similar effect on growing fibroblasts derived from patients with Hurler's syndrome (Danes \& Bearn, 1967). Within 1 month after the addition of retinol to the growing fibroblasts from an affected individual the uniform 'red rug' appearance of metachromatic staining mucopolysaccharides had disappeared and the characteristic ballooned 'gargoyle cells' in which the cells were distended with mucopolysaccharides were no longer visible. Within 6 weeks, it was no longer possible to distinguish the treated fibroblasts derived from patients with Hurler's syndrome from those obtained from normal subjects. If, however, the retinol was removed from the culture medium, the 'red rug' appearance was restored and 'gargoyle cells' reappeared.

Although the dramatic effect of retinol on mucopolysaccharide synthesis is not yet fully explained, it raises a point relative to the treatment not only of Hurler's syndrome, but also other inherited diseases. It was not so many years ago that the statement was made that 'the only cure for inherited diseases was the grave'. This is no longer true. The phenylalanine-free diet for phenylketonurics and galactose-free diet for galactosemics are but two examples where deprivation of the precursor of an enzymatic reaction may prevent harmful levels of a metabolite from accumulating in the body.

The effect of retinol on the fibroblasts derived from Hurler's syndrome suggests that another therapeutic approach may be useful. It seems likely that retinol is, in part, directly affecting the synthesis of cellular mucopolysaccharides. Thus, the phenotypic expression of the abnormal gene is being suppressed by a small repressor molecule, in this case retinol. Provided the suppressor molecule is not toxic it may well be possible to influence genetic disease in the future by controlling the expression of particular mutant genes. This approach seems more likely to yield clinically useful results in the next several decades than the heroic approaches of those who would assault the DNA by microlaser beams or by the induction of specific back mutations, methods that are not yet successful in the most simple of micro-organisms.

The results obtained have been encouraging, but they also underscore the constant need to 
return to the patient and determine to what extent the findings derived from in vitro studies are directly applicable to the human condition.

\footnotetext{
References

Danes, B.S. \& Bearn, A.G. (1966a) Hurler's syndrome. A genetic study in cell culture. $J$. exp. Med. 123, 1 .

DANES, B.S. \& BEARN, A.G. (1966b) Hurler's syndrome. Effect of retinol (vitamin A alcohol) on cellular mucopolysaccharides in cultured human skin fibroblasts. J. exp. Med. 124, 1181.
}

DANes, B.S. \& BEARN, A.G. (1967) The effect of retinol (vitamin A alcohol) on urinary excretion of mucopolysaccharides in the Hurler syndrome. Lancet, i, 1029.

Henderson, J.L. (1940) Gargoylism; review of principal features with report of five cases. Arch. Dis. Child. 15, 215.

McKusick, V.A. Heritable Disorders of Connective Tissue. Mosby, St Louis.

SAto, A. (1955) Chediak and Higashi's disease. Probable identity of 'a new leucocytal anomaly (Chediak)' and 'congenital gigantism of peroxidase granules (Higashi).' Tohoku J. exp. Med. 61, 201.

White, J.G. (1966) The Chediak-Higashi syndrome: A possible lysosomal disease. Blood, 28, 143.

\title{
Enteropoiesis: structural and functional relationships of the
}

\section{enterocyte}

\author{
C. C. Воотн
}

Royal Postgraduate Medical School, London

The Remarkable turnover of the cells of the intestinal mucosa was first suspected by Bizzozero as long ago as 1888 and has been firmly established by modern techniques during the past 20 years (Leblond \& Stevens, 1948; Leblond \& Messier, 1958 ; Creamer, Shorter \& Bamforth, 1961). The absorbing cells of the small intestine have, as Smyth has pointed out, a 'short life and a merry one ... if merriment is the equivalent in cellular terms of a remarkable range of activities possessed by few other cells in the body' (Smyth, 1967). Formed in the crypts of Lieberkuhn, the intestinal cells are extruded on to the surface of an adjacent villus whose side they climb until they reach the villus tip, from where they are shed into the intestinal lumen. The total turnover time for the cells of the jejunum is known to be as rapid as 2 or 3 days in the mouse and perhaps 5 days in man (MacDonald, Trier \& Everett, 1964 ; Shorter et al., 1964), so that the body only uses the extraordinary biological activities of these cells for a very brief period.

Doniach \& Shiner (1960) were the first to draw the analogy between the intestinal epithelial cells and the haemopoietic system. They compared the germinative or crypt zone, where the cells are manufactured, with the erythroblasts of the bone marrow and the adult absorbing cells of the villi with the erythrocytes of the peripheral blood. Doniach \& Shiner pointed out that in coeliac disease, where the jejunal mucosa is devoid of villi but the glandular or germinative layer of the small intestine is markedly increased, 'the situation is comparable with anaemia in which there is reduction of the peripheral red cells (villous cells) and compensatory hypertrophy of the red bone marrow (glandular mucosa)'.

This paper explores further the theoretical possibilities raised by Doniach's haematological analogy and suggests a new nomenclature for the intestinal mucosal cells. The functional implications of this classification are also discussed.

\section{Nomenclature of the intestinal absorbing cells}

There are a variety of different cell types present in the intestinal mucosa. Paneth cells, argentaffin cells and goblet cells mingle with the columnar cells which make up the major part of the absorptive epithelium and which are the concern of this paper. These columnar cells have been given a variety of names such as villous cells, absorptive cells, mucosal surface cells and adult epithelial cells, and the cells in the crypts of Lieberkuhn have been variously called the glandular cells, germinative zone or production zone. The use of a haematological analogy suggests a more satisfactory nomenclature which at the same time links the formative cells of the crypts with their adult counterparts. If the absorptive cell on the surface of the villus is given the term 'enterocyte', then the germinative cell in the glandular mucosa should be called an 'enteroblast', the function of this cell being 'enteropoiesis'. The further implications of this 